\title{
Sistemas de labranza y su incidencia sobre híbridos de maíz cultivado
}

\section{Tillage systems and their incidence on cultivated maize hybrids}

\author{
Juan Álvarez Arias ${ }^{1}$, Oscar Caicedo Camposano ${ }^{2,3 *}$, Carlos Salas Macías ${ }^{1}$ \\ ${ }^{1}$ Universidad Técnica de Manabí \\ 2 Universidad Técnica de Babahoyo \\ ${ }^{3}$ Universidad Nacional Agraria La Molina \\ *ocamposano@utb.edu.ec
}

DOI: https://doi.org/10.26871/killkanatecnica.v4i2.692

\begin{abstract}
Resumen
El propósito de este estudio fue establecer el efecto de tres sistemas de labranza y su incidencia sobre las propiedades físicas del suelo, la germinación, desarrollo y rendimiento del maíz duro amarillo en el cantón Babahoyo. Se utilizaron tres tratamientos de labranza: i) Labranza Convencional (LC); ii) Labranza mínima (LM) y iii) Labranza cero $\left(\mathrm{LC}_{0}\right)$ en donde se cultivaron tres híbridos de maíz. De los resultados obtenidos se concluye que los sistemas de labranza en términos aritméticos, produjeron cambios mínimos sobre las propiedades físicas del suelo (densidad aparente (Da), porosidad (P), resistencia mecánica (RM) e infiltración); observándose los cambios más consistentes prácticamente en la capa superficial con $\mathrm{LC}_{0}$, en estas mismas condiciones de labranza las raíces de los tres híbridos estudiados, alcanzaron un mayor volumen, altura de planta, peso del 1000 granos y rendimiento. Con respecto a la relación grano/tusa no se registraron diferencias estadísticamente significativas.
\end{abstract}

Palabras clave: Sistemas de labranza, suelo, maíz.

\begin{abstract}
The purpose of this research was to establish the effect of three tillage systems and their incidence on the physical properties of the soil, germination, development and yield of yellow hard maize in Babahoyo's canton. Three tillage treatments were used: i) Conventional tillage $(L C)$; ii) Minimum tillage (LM) and iii) Zero tillage (LC $\left.C_{0}\right)$ where three corn hybrids were grown. From the results obtained, it is concluded that tillage systems in arithmetic terms produced minimal changes on the physical properties of the soil (apparent density $(D a)$, porosity $(P)$, mechanical resistance (RM) and infiltration (I)). The germination of the seeds of the hybrids in the three tillage systems was carried out in a normal way; the most consistent changes happened in the superficial layer with $L C_{0}$ only, under these same tillage conditions, the roots of the three hybrids studied reached a higher volume, plant height, weight of 100 grains and yield. Regarding the grain/cob ratio, no statistically significant differences were recorded.
\end{abstract}

Keywords: Tillage systems, soil, maize.

\section{INTRODUCCIÓN}

En el Ecuador, la información sobre las prácticas de labranza utilizadas en el cultivo del maíz es escasa, particularmente en la zona agrícola de Babahoyo, perteneciente a la provincia de Los Ríos, Ecuador, se desconocen antecedentes de estudios que presenten los efectos de los sistemas de labranza sobre las propiedades físicas del suelo y su influencia en la germinación, desarrollo y rendimiento del maíz. La labranza superficial que por cultura se hace en el suelo para la siembra del maíz, en algunos casos en condiciones de humedad poco propicias, en un sistema de explotación altamente mecanizado, ha provocado que se manifiesten graves problemas de degradación física y que, a su vez, el desarrollo radicular de las plantas de maíz sea únicamente superficial [1].

Algunos académicos indican que el método de labranza escogido debe ser capaz de ajustarse considerablemente al sistema de manejo del suelo y desarrollo radicular de los cultivos, pensando siempre en conservar eficientemente los recursos suelo y agua [2] [3] [4].

En algunas latitudes del planeta mundo, se han realizado relacionadas los efectos de la labranza sobre las propiedades físicas del suelo y su incidencia en el comportamiento de varios cultivos de importancia económica y nutricional [5] [6] [7] [8] [9] [10]. Aunque algunas veces los resultados son contradictorios, muchos investigadores coinciden que 
en aquellos sistemas donde se perturba el suelo se consiguen generalmente mejores propiedades físicas (mayor macroporosidad y conductividad hidráulica, y menor densidad aparente) en la capa sometida a laboreo que en la mayoría de los sistemas conservacionistas. Sin embargo, el efecto de la labranza sobre dichos parámetros puede ser transitorio ya que, por impacto de las gotas de lluvia, el suelo puede recompactarse y disminuir la macroporosidad, lo cual tiene una influencia muy fuerte sobre el comportamiento del agua del suelo [11] [12] [13].

Las mejoras en la calidad del suelo ocurren con la preparación y adición de lodos de depuradora y abonos verdes, y los indicadores utilizados (densidad e infiltración de agua del suelo) fueron adecuados para la evaluación de estas mejoras [14].

El objetivo de esta investigación fue establecer el efecto de tres sistemas de labranza y su incidencia sobre las propiedades físicas del suelo, la germinación, desarrollo y rendimiento del maíz duro amarillo en el cantón Babahoyo.

\section{Metodología}

La presente investigación se realizó en los predios de propiedad del señor Rolando Jiménez ubicada en la parroquia Febrés Cordero del cantón Babahoyo en las coordenadas geográficas $1^{\circ} 51^{\prime} 24.5340$ ' de latitud sur, $79^{\circ} 26^{\prime}$ 13.1622" de longitud oeste; el sitio presenta temperatura máxima de $31.5^{\circ} \mathrm{C}$, temperatura mínima de $20.5^{\circ} \mathrm{C}$, precipitación anual de $2160 \mathrm{~mm}$ y 912 horas de brillo solar anuales [15].

Los tratamientos estuvieron, conformados por tres sistemas de labranza que; labranza convencional (LC), labranza mínima (LM) y labranza cero $\left(\mathrm{LC}_{0}\right)$, en esos suelos se cultivaron los híbridos de maíz duro amarillo de mayor demanda en el sector Dekalb 70881, Dekalb 7508 y Trueno NB7443 (La mención de marcas comerciales del material genético obedece únicamente a propósitos de identificación, no existiendo ningún compromiso promocional con relación a los mismos por la dirección de la revista), con los cuales se establecieron los tratamientos que se presentan en la Tabla I:

TABLA I: Tratamientos y subtratamientos

\begin{tabular}{lc}
\hline Sistemas de Labranza (Tratamientos) & Híbridos (Subtratamientos) \\
\hline & Trueno NB7443 \\
Labranza convencional & Dekalb 7508 \\
& Dekalb 7088 \\
\hline \multirow{2}{*}{ Labranza mínima } & Trueno NB7443 \\
& Dekalb 7508 \\
& Dekalb 7088 \\
\hline \multirow{2}{*}{ Labranza cero } & Trueno NB7443 \\
& Dekalb 7508 \\
& Dekalb 7088 \\
\hline
\end{tabular}

Se utilizó un diseño experimental de bloques completos al azar con arreglo en parcelas divididas con tres replicas, donde los tratamientos fueron tres sistemas de labranza, convencional (LC), mínima (LM) y cero $\left(\mathrm{LC}_{0}\right)$, los subtratamientos lo conformaron los tres híbridos que mayor demanda tienen en el mercado de semillas local como son el Dekalb 70881, Dekalb 7508 y Trueno NB7443, Para la comparación de las medias se utilizó la prueba de Tukey al $5 \%$ de probabilidad.

La labranza convencional consistió en dos pases del apero detallado en la Tabla II y la labranza mínima consistió en un pase del apero especificado en la Tabla 3; estos aperos contaron con la tracción de la maquinaria detallada en la
Tabla 1. Todas las labores de labranza se las realizaron tomando en cuenta las recomendaciones acerca de tempero de suelo realizadas por [16].

En la superficie del ensayo se tomaron muestras de suelo para su posterior análisis, estas muestras fueron llevadas al laboratorio de suelos de la Estación Experimental Tropical Sur del INIAP, para la determinación de parámetros físicos y químicos.

\section{A. Maquinaria empleada}

La maquinaria y los aperos utilizados para la preparación del suelo tanto en labranza convencional como en labranza mínima se detallan en las Tablas II, III y IV.

TABLA II: Datos técnicos de tractor utilizado T8 TIER 4B

\begin{tabular}{lclc}
\hline Características & Detalle & Características & Detalle \\
\hline 1. Rendimiento de motor & $275 \mathrm{kw}$ & 2. Dirección & Hidráulica \\
3. Neumáticos traseros & $710 / 70 \mathrm{R} 42$ & 4. Fabr. del motor & FPT \\
\hline
\end{tabular}


TABLA II: Datos técnicos de tractor utilizado T8 TIER 4B...continuación

\begin{tabular}{lclc}
\hline Características & Detalle & Características & Detalle \\
\hline 5. Neumáticos delanteros & $650 / 60 \mathrm{R} 34$ & 6. Par máximo & $1800 \mathrm{Nm}$ \\
7. Longitud de transporteN & $6.12 \mathrm{~m} \mathrm{~N}$ & 8. $\mathrm{N}^{\circ}$ de cilindrosN & 6 \\
9. Anchura transporte & $2.55 \mathrm{~m}$ & 10. Cilindro diámetro x carrera & $117 \times 135 \mathrm{~mm}$ \\
11. Altura de transporte & $3.375 \mathrm{~m}$ & 12. Revoluciones & $1400 \mathrm{rpm}$ \\
13. Velocidad & $50 \mathrm{~km} / \mathrm{h}$ & 14. Modelo de motor & Cursor 9 \\
15. Tipo de transmisión & $\mathrm{stfl.}$ & 16. Rendimiento de motor & $279 \mathrm{kw}$ \\
17. Peso & $12.45 \mathrm{t}$ & 18. Cilindrada & 8.71 \\
\hline
\end{tabular}

TABLA III: Implemento de labranza convencional CRSG

\begin{tabular}{lc}
\hline Características & Detalles \\
\hline Numero de discos & 20 \\
Diámetro de discos $(\mathrm{mm})$ & 711.2 \\
Diámetro del eje $(\mathrm{mm})$ & 41.3 \\
Ancho de trabajo $(\mathrm{mm})$ & 2250 \\
Espaciamiento entre discos $(\mathrm{mm})$ & 235 \\
Profundidad de trabajo $(\mathrm{mm})$ & $150-180$ \\
Peso aproximado $(\mathrm{kg})$ & 1853 \\
Tipo de ruedas & Simples \\
Potencia mínima del tractor $(\mathrm{kw})$ & 90 \\
\hline
\end{tabular}

TABLA IV: Implemento de labranza mínima

\begin{tabular}{lc}
\hline Características & Detalles \\
\hline Tipo de enganche & $\begin{array}{c}\text { De tiro } \\
\text { autonivelable }\end{array}$ \\
Número de timones $(\mathrm{mm})$ & 3 \\
Ancho del timón & 31.75 \\
Número de discos & 20 \\
Diámetro de discos & 609.6 \\
Ancho de trabajo $(\mathrm{mm})$ & 2200 \\
Número de posiciones & Variable \\
Angulo de ajuste en secciones $\left(^{\circ}\right)$ & $10-15$ \\
Máxima penetración $(\mathrm{mm})$ & 400 \\
Largo total (mm) & 6480 \\
Ancho del rodillo (mm) & 2200 \\
Rodillo desterronador & Estándar \\
Peso aproximado (kg) & 2010 \\
Caballaje mínimo del tractor $(\mathrm{kw})$ & $75-104$ \\
\hline
\end{tabular}

Los híbridos fueron sembrados de forma manual en una densidad de poblacional de 60000 plantas por hectárea, posteriormente se realizó las labores agrícolas necesarias como fertilización, la cual se realizará en función al análisis de suelo y necesidades nutricionales del cultivo, control de plagas previo monitoreo, se aplicaron productos agrotóxicos según los umbrales económicos y siguiendo las recomendaciones der las etiquetas de los mencionados productos, las necesidades hídricas del cultivo se satisficieron con riego por aspersión, se tomaba como referencia la capacidad de campo del suelo, ya que se ubicó un tensiómetro de 30 centímetros de longitud, de esta manera se evitó el estrés de las platas por exceso o déficit hídrico [17], la cosecha, se efectuó cuando los granos de maíz alcanzaron su madurez fisiológica.

\section{B. Variables evaluadas}

Contenido de humedad del suelo. - el contenido de humedad se determinó en laboratorio mediante el método termogravimétrico.

Resistencia mecánica. - La resistencia mecánica del suelo se registró al antes de la siembra y después de la cosecha, a las mismas profundidades de muestreo, el equipo que se utilizó fue un penetrómetro.

Densidad aparente. - Se tomaron muestras de suelo no disturbados con barrenos provisto de cilindros de volumen conocido a profundidades de $0-0.10 ; 0.10-0.20$ y $0.20-0.30$ metros de profundidad.

Porosidad. - luego de haberse determinado la densidad aparente y la densidad real del suelo, la porosidad se obtuvo por medio de la ecuación $\mathrm{P}=1$ - (Da/ Dr), donde Da es la densidad aparente y Dr es la densidad real del suelo.

Infiltración. - Se determinó por el método de los cilindros infiltrómetros, se realizaron tres pruebas con tres repeticiones sobre la superficie del ensayo.

Germinación. - Las evaluaciones se efectuaron a partir del tercer día después de la siembra, con una frecuencia de tres días hasta los 30 días que es cuando termina el proceso de germinación [18]

Volumen de raíces. - al final del ensayo, en cinco plantas de cada repetición se midió el volumen de raíces con una probeta de vidrio de $1000 \mathrm{~mL}$, a la que se aplicó agua hasta alcanzar un volumen de $500 \mathrm{~mL}$; en dicho volumen se sumergió el sistema radical lavado y picado, para registrar el volumen desplazado. Además, en igual número de plantas por repetición.[17] [19].

Altura de planta. - se midió desde el suelo hasta la base de la espiga; espués del estado lechoso del grano [20].

Relación grano/tusa. - se tomaron al azar 10 mazorcas por parcela experimental, éstas se desgranaron, y se procedió a pesar separadamente grano y tusa, estableciéndose la así la relación [21]. 
Peso de 100 granos. - se tomaron 100 granos o semillas por parcela experimental, se tuvo el cuidado de que los granos estén libres de daños de insectos y enfermedades; luego se procedió a pesar en una balanza de precisión [20].

Rendimiento. - estuvo determinado por el peso de los granos provenientes del área útil de cada parcela. El peso se ajustó al $14 \%$ de humedad y se transformó a toneladas por hectárea, se empleó la siguiente fórmula para ajustar los pesos $\mathrm{Pu}=((\mathrm{Pa}) *(100-\mathrm{ha}) /(100-$ hd)), donde Pu es el peso uniformizado; Pa peso actual; ha es humedad actual; hd es la humedad deseada.

\section{RESUlTAdOS}

Los resultados de algunas propiedades físicas que caracterizan inicialmente al suelo se muestran en la Tabla V. Se puede apreciar que la densidad aparente tiene un incremento en función a la profundidad, registrando un valor de 1,421 g.cm ${ }^{-3}$ entre los 20 y $30 \mathrm{~cm}$; además con este valor se ubica por encima del límite crítico señalado por [22], para esta clase textural. La porosidad del suelo determinada por una magnitud menor al valor crítico (10\%) y la resistencia mecánica, indican que podrían existir problemas de movimiento de agua en el perfil del suelo y de aireación en el mismo; en la Tabla VI también se presentan resultados de clase textural, materia orgánica nitrógeno $\mathrm{NH}_{4}$ Fosforo y Potasio, los cuales caracterizan también al suelo.
TABLA V: Características físicas del suelo

\begin{tabular}{lccc}
\hline Propiedades físicas & \multicolumn{3}{c}{ Profundidad $(\mathrm{cm})$} \\
\cline { 2 - 4 } & $0-10$ & $10-20$ & $20-30$ \\
\hline Densidad aparente $\left({\mathrm{g} . \mathrm{cm}^{-3}}^{-3}\right)$ & 1.312 & 1.403 & 1.421 \\
Porosidad (\%) & 41.226 & 40.207 & 37.413 \\
Resistencia Mecánica (psi) & 195,145 & 208.04 & 226.46 \\
\hline
\end{tabular}

TABLA VI: Características físico químicas del suelo

\begin{tabular}{lcc}
\hline Parámetros & & Profundidad 0-20 $(\mathrm{cm})$ \\
\hline Arena & $(\%)$ & 39 \\
Limo & $(\%)$ & 25 \\
Arcilla & - & 36 \\
Clase Textural & $(\%)$ & FA \\
Materia orgánica & $(\mu \mathrm{g} / \mathrm{ml})$ & 1.8 \\
Nitrógeno NH4 & $(\mu \mathrm{g} / \mathrm{ml})$ & 19 \\
Fosforo & $(\mu \mathrm{g} / \mathrm{ml})$ & 11 \\
Potasio & & 117 \\
\hline
\end{tabular}

En la Tabla VII se presentan los valores promedios de densidad aparente (Da), porosidad $(\mathrm{P})$, resistencia mecánica (RM) durante el desarrollo del cultivo. Estos datos indican que las condiciones del suelo al momento de la siembra fueron no diferentes como respuesta del mismo a los distintos tratamientos de labranza empleados. Los análisis de varianza de los parámetros antes mencionados no mostraron diferencias significativas $(\mathrm{p} \leq 0,05)$ para las profundidades estudiadas. Sin embargo, se notan mínimas diferencias aritméticas entre los momentos de muestreo en cada uno de los parámetros analizados, las cuales son favorables para el suelo.

TABLA VII: Valores promedios de densidad aparente (Da), porosidad (P), resistencia mecánica $(\mathrm{RM})$

\begin{tabular}{|c|c|c|c|c|c|c|c|}
\hline \multirow[t]{2}{*}{ Prof. (cm } & \multirow[b]{2}{*}{ Trat. } & \multicolumn{2}{|c|}{$\mathrm{Da}\left(\mathrm{g} . \mathrm{cm}^{-3}\right)$} & \multicolumn{2}{|c|}{$\mathrm{P}(\%)$} & \multicolumn{2}{|c|}{$\mathrm{RM}$ (psi) } \\
\hline & & MAS & MDC & MAS & MDC & MAS & MDC \\
\hline & LC & 1.312 & 1.311 & 41.156 & 41.226 & 195.145 & 194.833 \\
\hline \multirow{2}{*}{$0-10$} & $\mathrm{LC}_{0}$ & 1.321 & 1.320 & 37.349 & 37.413 & 226.460 & 226.098 \\
\hline & LC & 1.372 & 1.371 & 40.127 & 40.195 & 195.340 & 195.028 \\
\hline \multirow{2}{*}{$10-20$} & $\mathrm{LC}_{0}$ & 1.330 & 1.329 & 36.416 & 36.478 & 226.686 & 226.324 \\
\hline & $\mathrm{LC}$ & 1.357 & 1.356 & 38.975 & 39.041 & 195.535 & 195.223 \\
\hline \multirow[t]{2}{*}{$20-30$} & $\mathrm{LM}$ & 1.415 & 1.413 & 38.011 & 38.076 & 208.456 & 208.123 \\
\hline & $\mathrm{LC}_{0}$ & 1.392 & 1.391 & 35.370 & 35.390 & 226.913 & 226.750 \\
\hline
\end{tabular}

MAS: Muestra antes de la siembra; MDC: Muestra después de la cosecha.

La Tabla VIII muestra el desarrollo con el que se determinó el valor de infiltración básica antes de la siembra $4.737 \mathrm{~mm} \cdot \mathrm{h}^{-1}$, el mismo procedimiento se aplicó cuando se realizaron las pruebas de infiltración después de la cosecha, alcanzándose un valor de $4.745 \mathrm{~mm} \cdot \mathrm{h}^{-1}$.

En la Tabla IX se muestran los resultados obtenidos de las variables volumen de raíces, altura de planta, rela- ción grano/tusa, peso de cien granos y rendimiento; Los análisis de varianza de los parámetros antes mencionados reportaron diferencias significativas ( $\mathrm{p} \leq 0,05)$, los valores de volumen de raíces 34.24 cc y 34.10 cc obtenidos en labranza cero con hibrido DK7088 y DK 7508, se comportaron superiores e igual estadísticamente a los demás tratamientos. En el análisis de altura de planta se detectó 
que el híbrido Trueno NB7443 sembrado bajo los tres sistemas de labranza y el híbrido DK 7088 cultivado con labranza mínima y cero con valores de $193.03 \mathrm{~cm}, 10.06 \mathrm{~cm}$ y $192.91 \mathrm{~cm}$ respectivamente, se comportaron superiores e iguales al resto de los tratamientos.

TABLA VIII: Determinación de la infiltración básica

\begin{tabular}{|c|c|c|c|c|c|c|c|c|c|c|}
\hline 1 & 2 & 3 & 4 & 5 & 6 & 7 & 8 & & Ecuaciones & \\
\hline $\begin{array}{c}\text { Diferencia } \\
\text { de tiempo } \\
\quad(\min )\end{array}$ & $\begin{array}{c}\text { Tiempo } \\
\text { acumulado } \\
(\mathrm{min})\end{array}$ & $\begin{array}{c}\text { Lecturas } \\
\text { del nivel } \\
\text { de agua } \\
(\mathrm{mm})\end{array}$ & $\begin{array}{l}\text { Infiltración } \\
(\mathrm{mm})\end{array}$ & $\begin{array}{c}\text { Tasa de } \\
\text { Infiltración } \\
(\mathrm{mm} / \mathrm{min})\end{array}$ & $\begin{array}{c}\text { Tasa de } \\
\text { Infiltración } \\
(\mathrm{mm} / \mathrm{h})\end{array}$ & $\begin{array}{c}\text { Tasa de } \\
\text { Infiltración } \\
\text { acumulada } \\
(\mathrm{mm} / \mathrm{h})\end{array}$ & $\begin{array}{c}\text { Infiltración } \\
\text { acumulada } \\
\quad(\mathrm{mm})\end{array}$ & $\begin{array}{c}\text { Infiltración } \\
\text { Instantá- } \\
\text { nea } \\
(\mathrm{mm})\end{array}$ & $\begin{array}{l}\text { Infiltración } \\
\text { Promedio } \\
\text { (mm) }\end{array}$ & $\begin{array}{c}\text { Infiltración } \\
\text { Acumula- } \\
\text { da } \\
(\mathrm{mm})\end{array}$ \\
\hline 0 & 0 & 200,0 & 0,0 & 0,0 & 0,0 & 0,00 & 0,0 & 0,0 & 0,0 & 0,0 \\
\hline 2 & 2 & 199,8 & 0,2 & 0,10 & 6 & 6,00 & 0,2 & 0,11 & 0,12 & 0,23 \\
\hline 2 & 4 & 199,5 & 0,3 & 0,15 & 9 & 4,50 & 0,5 & 0,10 & 0,11 & 0,44 \\
\hline 2 & 6 & 199,3 & 0,2 & 0,10 & 6 & 2,00 & 0,7 & 0,10 & 0,11 & 0,64 \\
\hline 5 & 11 & 199,0 & 0,3 & 0,06 & 4 & 1,64 & 1,0 & 0,09 & 0,10 & 1,11 \\
\hline 5 & 16 & 198,5 & 0,5 & 0,10 & 6 & 1,88 & 1,5 & 0,09 & 0,10 & 1,55 \\
\hline 5 & 21 & 198,0 & 0,5 & 0,10 & 6 & 1,43 & 2,0 & 0,09 & 0,09 & 1,99 \\
\hline 10 & 31 & 197,0 & 1,0 & 0,10 & 6 & 1,94 & 3,0 & 0,08 & 0,09 & 2,84 \\
\hline 10 & 41 & 195,5 & 1,5 & 0,15 & 9 & 2,20 & 4,5 & 0,08 & 0,09 & 3,66 \\
\hline 10 & 51 & 195,0 & 0,5 & 0,05 & 3 & 0,59 & 5,0 & 0,08 & 0,09 & 4,46 \\
\hline 15 & 66 & 194,0 & 1,0 & 0,07 & 4 & 0,91 & 6,0 & 0,08 & 0,09 & 5,63 \\
\hline 15 & 81 & 193,5 & 0,5 & 0,03 & 2 & 0,37 & 6,5 & 0,08 & 0,08 & 6,79 \\
\hline 15 & 96 & 193,0 & 0,5 & 0,03 & 2 & 0,31 & 7,0 & 0,08 & 0,08 & 7,92 \\
\hline 15 & 111 & 192,0 & 1,0 & 0,07 & 4 & 0,54 & 8,0 & 0,07 & 0,08 & 9,04 \\
\hline
\end{tabular}

La comparación de las medias para la relación grano tusa no reporto diferencia entre los tratamientos; sin embargo, la variable peso de 100 granos reportó que el híbrido Trueno NB7443 sembrado bajo el sistema de labranza mínima y cero y el híbrido DK 7088 cultivado bajo las mismas con- diciones obtuvieron valores de $42.35 \mathrm{~g}, 43.12 \mathrm{~g}, 42.66 \mathrm{~g}$, y $43.98 \mathrm{~g}$ respectivamente, los mismos que fueron superiores e iguales a los demás valores correspondientes a los otros tratamientos.

TABLA IX: Variables agronómicas evaluadas

\begin{tabular}{|c|c|c|c|c|c|}
\hline $\begin{array}{ll}\text { Variables } & \text { Tratamientos } \\
\end{array}$ & VR (cm3) & $\mathrm{AP}(\mathrm{cm})$ & RGT & PCG (g) & $\mathrm{R}\left(\mathrm{t} \cdot \mathrm{ha}^{-1}\right)$ \\
\hline LC - H1 & $31.41 \mathrm{ab}$ & $191.92 \mathrm{a}$ & 80.57 & $40.98 \mathrm{~b}$ & $10.55 \mathrm{~b}$ \\
\hline LM - H1 & $32.39 \mathrm{ab}$ & $192.61 \mathrm{a}$ & 80.83 & $42.35 \mathrm{a}$ & $11.02 \mathrm{a}$ \\
\hline $\mathrm{LC}_{0}-\mathrm{H} 1$ & $33.03 \mathrm{ab}$ & $193.03 \mathrm{a}$ & 80.76 & $43.12 \mathrm{a}$ & $11.34 \mathrm{a}$ \\
\hline $\mathrm{LC}-\mathrm{H} 2$ & $31.39 \mathrm{ab}$ & $184.44 \mathrm{ab}$ & 80.54 & $39.39 \mathrm{~b}$ & $9.90 \mathrm{~b}$ \\
\hline LM - H2 & $31.24 \mathrm{ab}$ & $187.27 \mathrm{ab}$ & 80.55 & $39.43 \mathrm{~b}$ & $9.90 \mathrm{~b}$ \\
\hline $\mathrm{LC}_{0}-\mathrm{H} 2$ & $34.10 \mathrm{a}$ & $188.12 \mathrm{ab}$ & 80.62 & $42.89 \mathrm{~b}$ & $10.68 \mathrm{ab}$ \\
\hline LC - H3 & $30.91 \mathrm{ab}$ & $187.23 \mathrm{ab}$ & 80.45 & $39.55 \mathrm{~b}$ & $7.92 \mathrm{~b}$ \\
\hline LM - H3 & $31.43 \mathrm{ab}$ & $190.06 \mathrm{a}$ & 80.60 & $42.66 \mathrm{a}$ & $8.19 \mathrm{~b}$ \\
\hline $\mathrm{LC}_{0}-\mathrm{H} 3$ & $34.24 \mathrm{a}$ & $192.91 \mathrm{a}$ & 80.53 & $43.98 \mathrm{a}$ & $11.71 \mathrm{a}$ \\
\hline
\end{tabular}

VR: Volumen de raíces; AP: Altura de Plantas; RGT: Relación grano/tusa; PCG: Peso del 1000 granos; R: Rendimiento

De las observaciones y procesamientos realizadas para la variable rendimiento se obtuvo que el híbrido Trueno NB7443 cultivado con labranza mínima y cero y que el híbrido DK 7088 sembrado con labranza cero, alcanzaron cifras de $11.02,11.34$ y 11.71 se comportaron superiores estadísticamente e iguales estadísticamente al promedio del tratamiento DK 7508 cultivado con labranza cero.

\section{CONCLUSIONES}

En términos absolutos, las labores de labranza provocaron un efecto modificante no significativo de las propieda- 
des físicas el suelo labrado, la cual depende del implemento y de la profundidad de laboreo del mismo; la infiltración aumento en poco menos de una unidad, esto debido al ligero aumento en la porosidad del suelo arado con labranzas conservacionistas.

Las modificaciones observadas al final del ensayo podrían presentarse en mayor magnitud al realizarse más de una campaña en las que prevalezca la labranza cero, ya que es el tiempo y entre otras las prácticas de labranza conservacionistas las que potencializan la resiliencia del suelo.

\section{RECOMENDACIONES}

Realizar estudios posteriores bajo las mismas condiciones de labranza conservacionista y manejo del cultivo de maíz, con la finalidad de medir de manera temporal la resiliencia de las propiedades físicas del suelo y la respuesta agronómica del maíz.

\section{REFERENCIAS}

[1] [1] I. D. N. Espinoza, M. D. C. Zenteno, J. C. Chávez, V. N. Moreiral, K. E. A. Solarte, y F. L. M. Intriago, «Propiedades físicas del suelo en diferentes sistemas agrícolas en la provincia de Los Ríos, Ecuador», Temas agrarios, vol. 23, n.o 2, pp. 177-187, 2018.

[2] [2] W. L. Harman, G. J. Michels, y A. F. Wiese, «A conservation tillage system for profitable cotton production in the Central Texas High Plains», Agronomy Journal, vol. 81, n.o 4, pp. 615-618, 1989.

[3] [3] L. R. P. Serrano, J. L. H. Martos, y V. S.-G. Renedo, «Influencia de cuatro sistemas de laboreo en las propiedades físicas de un Fluvisol y en el balance energético en cultivos de raíces y tubérculos», Universidad\&Ciencia, vol. 6, n.o 1, pp. 65-81, 2017.

[4] [4] M. A. Busari, S. S. Kukal, A. Kaur, R. Bhatt, y A. A. Dulazi, «Conservation tillage impacts on soil, crop and the environment», International Soil and Water Conservation Research, vol. 3, n.o 2, pp. 119-129, 2015.

[5] [5] B. P. Cadena, D. Egas, M. H. R. Eraso, J. Mosquera, y O. Benavides, «Efecto de cinco sistemas de labranza, en la erosión de un suelo vitric haplustand, bajo cultivo de papa (Solanum tuberosum L)», Revista de Ciencias Agrícolas, vol. 29, n.o 2, pp. 116-128, 2012.

[6] [6] R. Lal, «Importance of tillage systems in soil and water management in the tropics». IITA Proc. Ser., 1979.

[7] [7] S. M. Brown, T. Whitwell, J. T. Touchton, y C. H. Burmester, «Conservation tillage systems for cotton production», Soil Science Society of America Journal, vol. 49, n.o 5, pp. 1256-1260, 1985.

[8] [8] N. Gómez-Calderón, K. Villagra-Mendoza, y M. Solorzano-Quintana, «La labranza mecanizada y su impacto en la conservación del suelo (revisión literaria)», Revista Tecnología en Marcha, vol. 31, n.o 1, pp. 167-177, 2018.
[9] [9] M. S. Vilche y C. Alzugaray, «Efecto de la labranza profunda sobre el rendimiento y uso del agua del cultivo de maíz.», Revista de Investigaciones de la Facultad de Ciencias Agrarias-UNR, n.o 14, pp. 011-025, 2016.

[10] [10] C. Bravo, «La labranza conservacionista en Venezuela. III Reunión de la Red Latinoamericana de labranza conservacionista (RELACO)», San José, Costa Rica. Memorias, pp. 235-247, 1995.

[11] [11] C. Bravo y A. Florentino, «Efecto de diferentes sistemas de labranza sobre las propiedades físicas del suelo y su influencia sobre el rendimiento del algodón», Bioagro, vol. 9, n.o 3, pp. 67-75, 1997.

[12] [12] R. García, «Efecto de sistemas de labranza en propiedades físicas del suelo y desarrollo radicular del cultivo de quinua (Chenopodium quinoa W.)», Revista del Instituto de Investigación de la Facultad de Ingeniería Geológica, Minera, Metalúrgica y Geográfica, vol. 18, n.o 35, pp. 109-113, 2016.

[13] [13] J. Herrera-Puebla, «Efecto de dos sistemas de labranza sobre la infiltración en suelos Ferralíticos Rojos», Revista Ingeniería Agrícola, vol. 7, n.o 4, pp. 3-10, 2017.

[14] [14] M. C. Alves, L. G. A. S. Suzuki, y L. E. A. S. Suzuki, «Densidade do solo e infiltração de água como indicadores da qualidade física de um Latossolo Vermelho distrófico em recuperação», Revista Brasileira de Ciência do Solo, vol. 31, n.o 4, pp. 617-625, 2007 , doi: 10.1590/S0100-06832007000400002.

[15] [15] INAMHI, «Registros climáticos históricos de la Estación INAMHI - UTB.» Instituto Nacional de Meteorología e Hidrología, 2020.

[16] [16] Y. E. Olivet-Rodríguez y D. Cobas-Hernández, «Efecto de dos sistemas de labranza mínima sobre la porosidad de un fluvisol para cultivo del frijol (phaseolus vulgaris 1.)(original)», REDEL. Revista Granmense de Desarrollo Local, vol. 1, n.o 1, pp. 13-21, 2017.

[17] [17] O. Caicedo-Camposano, D. Cadena-Piedrahita, E. Galarza-Centeno, y D. Solorzano-Galarza, «Permisibilidad del maíz (Zea mays L.) sometido a diferentes condiciones de inundación: Determinación del tiempo de drenaje en Babahoyo, Ecuador», Revista Científica y Tecnológica UPSE, vol. 6, n.o 2, pp. 67-75, 2019.

[18] [18] O. Caicedo-Camposano, D. Cadena-Piedrahita, M. Goyes-Cabezas, y N. Rojas-Jorgge, «Influence of seed planting depth on germination and physiological development of seedlings of maize (Zea mays L.)», Killkana Técnica, vol. 4, n.o 1, Art. n.o 1, abr. 2020, doi: 10.26871/killkana_tecnica.v4i1.590.

[19] [19] W. C. Quintal Ortiz, A. Pérez-Gutiérrez, L. Latournerie Moreno, C. May-Lara, E. Ruiz Sánchez, y A. J. Martínez Chacón, «Uso de agua, potencial hídrico y rendimiento de chile habanero (Capsicum chinense Jacq.)», Revista fitotecnia mexicana, vol. 35, n.o 2, pp. 155-160, 2012.

[20] [20] CIMMYT, Descriptors for Maize. International Maize and Wheat Improvement Center, Mexico 
City/International Board for Plant Genetic Resources.

Recibido: 20 de febrero de 2020 Mexico: CIMMYT, 1991.

[21] [21] J. L. Arellano Vázquez, J. Virgen Vargas, I. Rojas Martínez, y M. A. Avila Perches, «H-70: Híbrido de maíz de alto rendimiento para temporal y riego del Altiplano Central de México», Revista mexicana de ciencias agrícolas, vol. 2, n.o 4, pp. 619-626, 2011.

[22] [22] I. Pla, «Metodología para la caracterización física con fines de diagnóstico de problemas de manejo y conservación de suelos en condiciones tropicales.», Revista Facultad de Agronomía, U.C.V. Maracay., vol. Avance $\mathrm{N}^{\circ}$ 30, 1983.

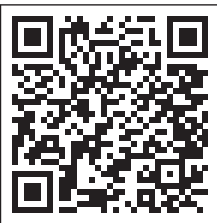


